\title{
Richard JONES
}

Historien, professeur retraité, département d'histoire, Université Laval

(1983)

\section{Duplessis}

et le gouvernement

de l’Union nationale

\author{
Un document produit en version numérique par Jean-Marie Tremblay, bénévole, \\ professeur de sociologie au Cégep de Chicoutimi \\ Courriel: jean-marie_tremblay@uqac.ca \\ Site web pédagogique : http://www.uqac.ca/jmt-sociologue/ \\ Dans le cadre de: "Les classiques des sciences sociales" \\ Une bibliothèque numérique fondée et dirigée par Jean-Marie Tremblay, \\ professeur de sociologie au Cégep de Chicoutimi \\ Site web: http://classiques.uqac.ca/ \\ Une collection développée en collaboration avec la Bibliothèque \\ Paul-Émile-Boulet de l'Université du Québec à Chicoutimi \\ Site web: http://bibliotheque.uqac.ca/
}




\section{Politique d'utilisation de la bibliothèque des Classiques}

Toute reproduction et rediffusion de nos fichiers est interdite, même avec la mention de leur provenance, sans l'autorisation formelle, écrite, du fondateur des Classiques des sciences sociales, Jean-Marie Tremblay, sociologue.

Les fichiers des Classiques des sciences sociales ne peuvent sans autorisation formelle:

- être hébergés (en fichier ou page web, en totalité ou en partie) sur un serveur autre que celui des Classiques.

- servir de base de travail à un autre fichier modifié ensuite par tout autre moyen (couleur, police, mise en page, extraits, support, etc...),

Les fichiers (.html, .doc, .pdf, .rtf, .jpg, .gif) disponibles sur le site Les Classiques des sciences sociales sont la propriété des Classiques des sciences sociales, un organisme à but non lucratif composé exclusivement de bénévoles.

Ils sont disponibles pour une utilisation intellectuelle et personnelle et, en aucun cas, commerciale. Toute utilisation à des fins commerciales des fichiers sur ce site est strictement interdite et toute rediffusion est également strictement interdite.

L'accès à notre travail est libre et gratuit à tous les utilisateurs. C'est notre mission.

Jean-Marie Tremblay, sociologue

Fondateur et Président-directeur général, LES CLASSIQUES DES SCIENCES SOCIALES. 
Cette édition électronique a été réalisée par Jean-Marie Tremblay, bénévole, professeur de sociologie au Cégep de Chicoutimi à partir de :

\section{Richard Jones}

\section{Duplessis et le gouvernement de l’Union nationale.}

Ottawa, La Société Historique du Canada, 1983, 23 pp. Brochure historique no 35.

[Autorisation formelle accordée par l'auteur le 27 septembre 2008 de diffuser toutes ses publications dans Les Classiques des sciences sociales.]

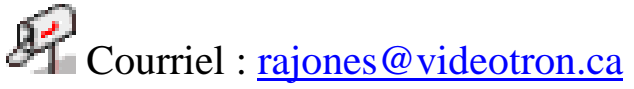

Polices de caractères utilisée :

Pour le texte: Times New Roman, 14 points.

Pour les notes de bas de page : Times New Roman, 12 points.

Édition électronique réalisée avec le traitement de textes Microsoft Word 2008 pour Macintosh.

Mise en page sur papier format : LETTRE US, 8.5'’ x 11',

Édition numérique réalisée le 2 septembre 2012 à Chicoutimi, Ville de Saguenay, Québec.

\section{Fait avec}

Macintosh 


\title{
Richard JONES
}

Historien, professeur retraité, département d’histoire, Université Laval

\author{
Duplessis \\ et le gouvernement de l’Union nationale
}

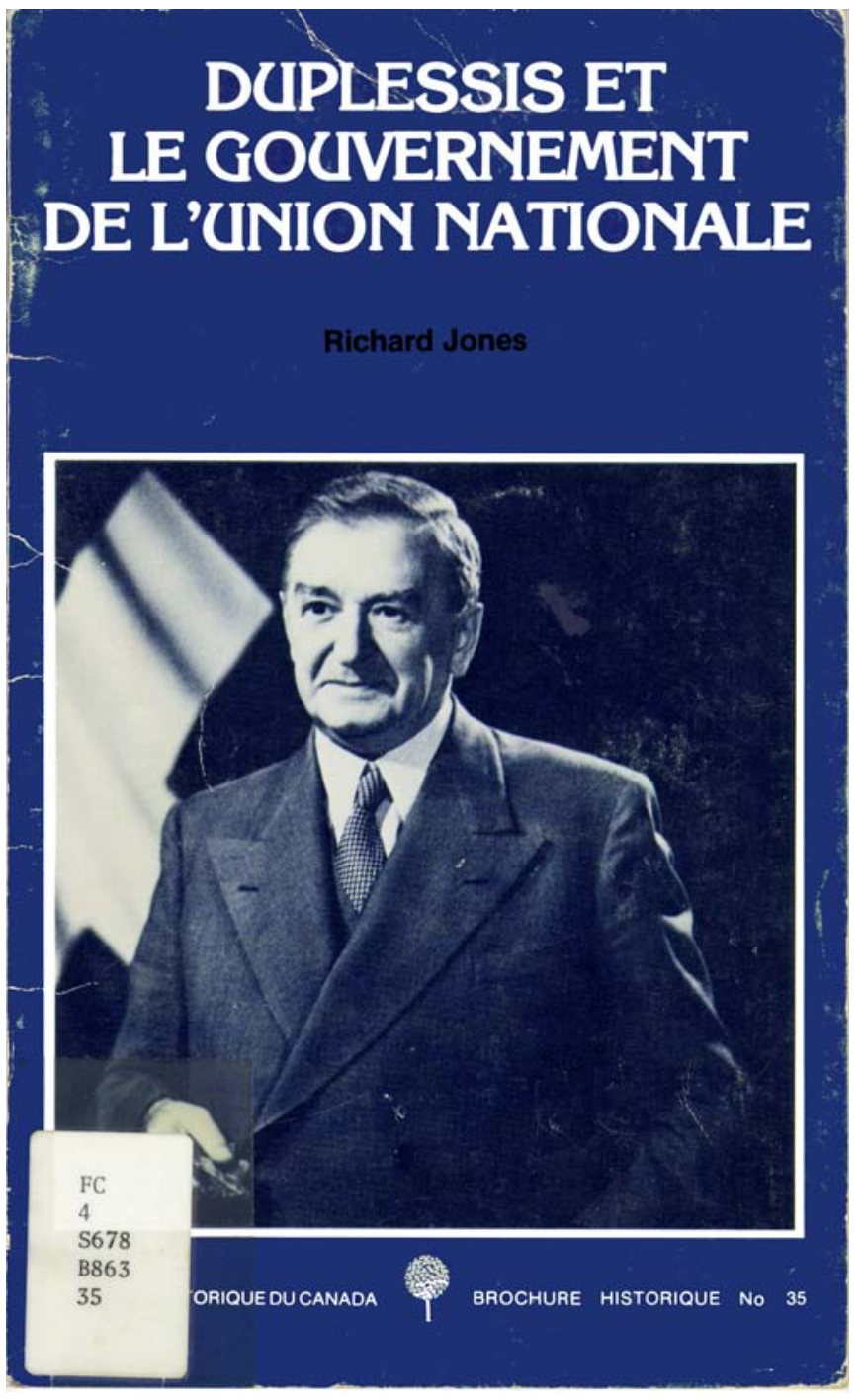

Ottawa, La Société Historique du Canada, 1983, 23 pp. Brochure historique no 35. 


\section{Table des matières}

$\underline{\text { Richard Jones }}$

Duplessis et le gouvernement de l'Union nationale

I. Vers le pouvoir

II. Premier mandat, 1936-1939

III. Séjour dans l'opposition, 1939-1944

IV. Les assises du pouvoir

V. Le duplessisme contesté

Notes bibliographiques 
[2]

$\underline{\text { Retour à la table des matières }}$

Richard Jones est né aux États-Unis et, en 1948, s'est établi, avec sa famille, à Cooksville (Mississauga), Ontario, où il a fait ses études primaires et secondaires. Il est diplômé des universités Princeton et Laval. Après avoir enseigné l'histoire au Collège Sainte-Anne-de-laPocatière de 1966 à 1968, il est entré à l'Université Laval en 1970 comme professeur d'histoire canadienne. En 1977, il a été professeur invité au Centre d'études canadiennes de l'Université Johns Hopkins, à Washington, D.C. et, en 1983, chercheur invité au Centre d'études canadiennes de l'Université Duke, à Durham, Caroline du Nord. Il s'intéresse particulièrement à l'histoire politique et culturelle du Canada et du Québec au vingtième siècle ainsi qu'aux libertés civiles et aux droits des minorités. Parmi ses principaux ouvrages on retient : Community in Crisis : French-Canadian Nationalism in Perspective (Toronto, 1967, 1972), L'Idéologie de "Action catholique" (1917-1939) (Québec, 1974), Histoire du Québec (Toulouse et St-Hyacinthe, 1976, en collaboration), et Vers une hégémonie libérale : Aperçu de la politique canadienne de Laurier à King (Québec, 1980). 
[3]

\section{DUPLESSIS ET LE GOUVERNEMENT DE L'UNION NATIONALE}

$\underline{\text { Retour à la table des matières }}$

Personnage haut en couleur, Maurice Le Noblet Duplessis fut premier ministre du Québec de 1936 à 1939 et à nouveau de 1944 jusqu'à sa mort en 1959. Quoique d'un physique assez ordinaire, il était doté d'un nez allongé qui faisait la joie des caricaturistes. Préférant les habits sobres et de bon goût, on le voyait toutefois coiffé d'un vieux chapeau afin, disait-il, de se "rapprocher du peuple". Homme intelligent et possédant la repartie facile, il n'était nullement un intellectuel et, d'ailleurs, se targuait d'avoir les deux pieds à terre. Sa vie privée fut plutôt austère, du moins après son retour au pouvoir en 1944, et il avait peu de distractions, mis à part la musique et le baseball. En effet, la politique l'habitait et, célibataire, il se disait marié à sa province.

Peu d'hommes politiques canadiens depuis la Confédération ont suscité autant de controverses que Duplessis. Adulé par ses partisans, honni par ses adversaires, Duplessis a dominé la scène politique québécoise durant près d'un quart de siècle. Après sa mort, d'autres ont voulu créer un Québec nouveau, un Québec moderne, libéré de ce qu'ils percevaient comme les entraves du duplessisme, et de nombreux commentateurs, partageant les objectifs du nouveau gouvernement libéral de Jean Lesage, ont peint un tableau sombre des années de 
gouvernement unioniste. Comme l'histoire, toutefois, ne porte jamais de jugements définitifs, d'autres observateurs de cette période ont voulu, dès les années 1970, étudier Duplessis sous un angle plus impartial sinon avec une certaine sympathie.

Quiconque poursuit une réflexion à propos de Duplessis est confronté à des interprétations diverses et même contradictoires au sujet de ce personnage. Bien qu'il se vît humilié quotidiennement à l'Assemblée législative par le premier ministre, Georges-Émile Lapalme, chef de l'opposition libérale de 1950 à 1958, ne cache pas son admiration pour cet homme qui "ressentait les vibrations populaires, comme s'il eût plaqué un stéthoscope". Le Père Georges-Henri Lévesque, ancien doyen de la Faculté des Sciences sociales à l'Université Laval et critique acerbe du gouvernement unioniste, estime que Duplessis possédait "une habileté vraiment exceptionnelle, une lucidité extraordinaire" mais qu'il fut en même temps "un très petit politicien, parce que presque tous ses jugements, presque toutes ses actions, étaient mesquins sur le plan politique". Les biographes sont aussi partagés dans les jugements qu'ils portent sur Duplessis et son régime. Pour le journaliste Leslie Roberts, celui qu'il appelle "le chef" avait pour mobile essentiel "une soif avide de pouvoir" ; pour le garder, il y allait avec la même absence de vergogne qu'un dictateur sudaméricain". L'historien-prêtre Lionel Groulx affirme dans ses Mémoires ne s'être jamais payé d'illusions sur le personnage. Pierre Laporte, alors chroniqueur [4] au journal Le Devoir, trouve Duplessis généreux, mais "d'une incroyable mesquinerie envers les gens qu'il n'aimait pas" ; ce fut un parlementaire "haut en couleur, captivant... mais n'ayant aucun respect pour les principes démocratiques". L'historien Robert Rumilly, par contre, ne tarit pas d'éloges pour cet homme qui a toujours entretenu un "profond amour de sa province et de son peuple", qui a eu "le sens national, l'instinct des intérêts québécois". Manifestement, si tous parlent du même individu, tous ne voient pas le même homme.

Certes, le succès électoral remarquable de Duplessis, mise à part la déroute de 1939, ne fait pas de doute. Mais dès que l'historien de cette période se met à approfondir son analyse du régime, il se bute à des problèmes d'interprétation de taille. Duplessis a-t-il vraiment été un dictateur corrompu qui ne se souciait que du sort de son parti et de sa propre place en tant que chef ? Ou faut-il plutôt le voir comme le lea- 
der de son peuple, exprimant ses désirs, incarnant ses volontés, défendant ses intérêts ? Doit-on conclure, comme plusieurs l'ont prétendu, qu'il a "retardé" l'évolution du Québec, ralenti l'avènement d'une "société moderne ?" Ou n'a-t-il pas, par contre, compris que le Canada français formait une société distincte qu'il fallait préserver devant les assauts inlassables d'adversaires qui voulaient la détruire? Il reste, cependant, que ce personnage avait de multiples facettes, et selon celles privilégiées par les historiens comme par les autres auteurs qui l'ont étudié, le portrait de l'homme et du régime varie grandement.

\section{Vers le pouvoir}

\section{$\underline{\text { Retour à la table des matières }}$}

Que Maurice Duplessis soit entré en politique provinciale comme un conservateur, membre d'un parti exilé dans le désert politique au Québec depuis 1897 et montrant alors fort peu de signes d'une résurgence, s'explique largement par tradition familiale. Son père, Nérée, n'a-t-il pas été député conservateur à l'Assemblée législative de 1886 à 1900 ? Rappelons aussi, pour comprendre les relations futures entre Duplessis et l'Église, que Trois-Rivières, où Duplessis a été élevé, était le siège du diocèse de Mgr Louis-François Laflèche et donc un bastion d'ultramontanisme et de conservatisme.

Duplessis sera le type de politicien, attentif aux besoins et aux préoccupations de ses électeurs, qui fera après 1944 la force de l'Union nationale. Dès sa première campagne électorale, celle de 1923 qu'il a d'ailleurs perdue, Duplessis privilégie le rapport avec l'électeur, et tous les événements locaux sortant un peu de l'ordinaire - naissances, baptêmes, mariages, décès, prix, etc. - font l'objet de lettres de félicitations ou de condoléances selon le cas. Un tel rapport est possible dans les campagnes ou dans les petites villes où se trouvent relativement peu de votants [5] - Trois-Rivières ne compte qu'environ 5,000 électeurs en 1927 et la moyenne des circonscriptions dans la province n'en renferme que 6,700. Mais trente ans plus tard, alors qu'on dénombre 26,000 inscrits par district et que des politiques de plus grande envergure se substituent dans une certaine mesure à des questions d'intérêt purement local, ce genre de rapport personnalisé devient, sauf dans les 
comtés ruraux à faible population, beaucoup plus difficile à pratiquer. L'Union nationale en souffrira grandement.

Lorsqu'en 1927 Duplessis est élu pour la première fois député de Trois-Rivières, il fait partie d'une petite phalange de 9 députés conservateurs dans une Chambre dominée par 75 libéraux habitués au pouvoir. Sous le leadership d'Arthur Sauvé, l'opposition est en nette régression (elle avait fait élire 19 députés en 1923) et ravagée par des dissensions internes. Camillien Houde, alors maire de Montréal, remplace Sauvé en 1929 mais il perd son propre siège aux élections de 1931 même si les conservateurs réussissent à augmenter leur proportion des suffrages de près de $8 \%$. De plus en plus contesté au sein de son parti, Houde remet sa démission en septembre 1932 et Duplessis est choisi par le caucus comme chef parlementaire de l'opposition.

Dans son nouveau rôle, Duplessis fait valoir un certain nationalisme économique et attaque les puissances de l'argent qu'il dit inféodées au parti libéral. Duplessis réclame aussi le crédit agricole provincial et il évoque le credo qu'il respectera quand il deviendra lui-même premier ministre : lors d'un discours prononcé en Chambre au début de 1933, il engage le gouvernement à "se rendre à l'évidence et [à] reconnaître, dans sa législation et dans ses actes, que la province de Québec a toujours été et doit toujours être essentiellement agricole". Pourtant, d'après le recensement de 1931, 65\% des citoyens de la province habitent déjà les villes !

Au congrès à la chefferie tenu à Sherbrooke en octobre 1933, Duplessis défait sans mal (332 à 214 voix) Onésime Gagnon, député conservateur aux Communes d'Ottawa, qui favorisait des liens plus proches avec l'aile fédérale et "le chef suprême, R.B. Bennett". Faut-il voir dans cette victoire les premiers jalons de l'autonomisme dont Duplessis fera montre plus tard alors qu'il se targuera d'avoir établi un parti provincial libre de toutes attaches à un parti fédéral ? Certes, Duplessis sent l'impopularité grandissante du premier ministre canadien, mais il propose lui-même une résolution d'appui, quoiqu'en des termes édulcorés, au chef fédéral. S'il triomphe à Sherbrooke, c'est sans doute principalement à cause d'une organisation supérieure à celle de son rival, non pas en raison de prises de position nationalistes.

Alors que le Québec est plongé en pleine crise économique, de nombreux esprits se mettent à la recherche de solutions qui, sans ver- 
ser dans le [6] socialisme, permettront de corriger les pires abus du régime capitaliste. Sous l'égide de l'École sociale populaire, organisme dirigé par les Jésuites, un groupe de laïcs catholiques bien connus, incluant entre autres Albert Rioux, de l'Union catholique des cultivateurs, Wilfrid Guérin, des caisses populaires, et Alfred Charpentier, des syndicats catholiques, rédige, en 1933, le "Programme de Restauration sociale". Ce programme suscitera un vif intérêt chez certains libéraux, dont Paul Gouin, fils de l'ancien premier ministre du Québec, Lomer Gouin. En 1934, ceux-ci forment un nouveau parti politique, l'Action libérale nationale, en s'abouchant à des nationalistes à l'esprit réformiste comme Philippe Hamel, Ernest Grégoire et René Chaloult. Leur programme, qui s'inspire des principes du Programme de Restauration sociale, promet une lutte vigoureuse contre les trusts, notamment celui de l'électricité, un assainissement des moeurs électorales, la création d'un ministère du Commerce, et l'adoption de réformes ouvrières bien qu'il n'y soit pas question de syndicats. Toutefois, le premier article de ce programme touche les réformes agraires et les auteurs soutiennent que "l'oeuvre de restauration économique se ramène principalement à une oeuvre de restauration rurale, basée sur l'agriculture familiale et la coopération".

Le premier ministre et chef libéral Louis-Alexandre Taschereau, alors à l'apogée de son pouvoir et habitué au bipartisme qu'il sait contrôler, ne voit évidemment pas d'un bon oeil la fondation d'un tiers parti à la veille des élections de 1935 ; Maurice Duplessis, chef du parti conservateur, n'est guère plus enthousiaste. Quelle chance une opposition divisée aura-t-elle de renverser un régime solidement ancre au pouvoir ? Il ne faut donc pas se surprendre de ce que Duplessis, s'il dénonce Taschereau pour avoir favorisé les étrangers, négligé les cultivateurs et miné les traditions et la religion, ne ménage pas Gouin qu'il considère comme un travailleur de la onzième heure, et promette de présenter des candidats conservateurs dans tous les districts. De leur côté, les leaders de l'A.L.N. se méfient beaucoup de Duplessis, le jugeant opportuniste et trop ambivalent notamment en ce qui concerne la politique à appliquer face au trust de l'électricité, tant décrié par Philippe Hamel en particulier. Toutefois, les circonstances conduiront à un mariage de convenance entre les deux partis oppositionnistes, rapprochement dont ni Gouin ni Duplessis ne prendront vraiment l'initiative. 
Il semble clair, en effet, que les militants de la base des deux formations politiques poussent les chefs vers une alliance. Malgré les ressources oratoires importantes de l'A.L.N. et l'expérience parlementaire des conservateurs, les deux partis manquent d'argent pour faire une campagne efficace contre Taschereau. Enfin, à une réunion orageuse entre les [7] principaux porte-parole de chaque parti, en l'absence de Duplessis lui-même, on s'entend sur la répartition des comtés entre candidats conservateurs et candidats de l'A.L.N. aussi bien que sur le leadership de la nouvelle coalition. Ainsi, les accords prévoient qu'aux prochaines élections, de 25 à 30 comtés seront réservés aux conservateurs et une soixantaine à l'A.L.N., et qu'après la victoire Maurice Duplessis deviendra premier ministre alors que Paul Gouin désignera la majorité des ministres. Malgré l'avantage apparent de l'A.L.N. dans le partage des candidatures, nombre des candidats de ce parti sont en réalité des conservateurs qui croient mieux réussir sous la bannière de l'A.L.N. Le 7 novembre 1935, donc, Duplessis et Gouin annoncent publiquement la formation de "L'Union nationale Duplessis-Gouin" en vue de présenter un "front uni contre l'ennemi du peuple de la province de Québec : le régime Taschereau". Son programme officiel est celui de l'A.L.N. Aux élections du 25 novembre, le régime libéral est fortement ébranlé : 48 libéraux sont élus contre 42 oppositionnistes. De ces derniers, 16 sont officiellement conservateurs et 26, membres de l'A.L.N.

Les événements des mois suivants confirment Duplessis comme seul chef de l'Union nationale. Pour ses adversaires, le chef conservateur s'est ingénié à miner la position de Paul Gouin au sein de l'Union nationale avant de se débarrasser, une fois devenu premier ministre, des autres porte-parole trop réformistes de l'A.L.N., et finalement, d'écarter les aspects les plus radicaux du programme, notamment la lutte contre la dictature économique. Quoi qu'il en soit, la plupart des observateurs jugent que Gouin n'avait vraiment pas l'étoffe d'un chef, que c'était un intellectuel qui n'était nullement rompu à la politique quotidienne. Par ailleurs, il est vrai que Duplessis s'est employé à renforcer sa position auprès des membres de l'A.L.N. A ce sujet, le secrétaire parlementaire de ce parti confirmera plus tard : "Dans l'esprit de nos députés, dans les faits aussi, ils ont vu que le seul homme capable de les mener, de les conduire, était réellement Maurice Duplessis". Ce 
leadership va se démontrer avec vigueur lors des célèbres enquêtes du Comité des comptes publics.

Il se peut qu'il s'agisse alors d'une "bagatelle ridicule", comme le prétendra plus tard le juriste Jean-Charles Bonenfant, et que les prétendus scandales du gouvernement Taschereau n'étaient que des peccadilles. Mais il reste que les témoignages entendus au cours des séances du Comité, séances que Maurice Duplessis dirige personnellement, suscitent un intérêt immense et réussissent à discréditer le régime. Que le ministre Irénée Vautrin ait enfilé des culottes payées par l'État avant d'effectuer des randonnées en forêt n'a peut-être pas en soi beaucoup d'importance, mais le cri des "culottes à Vautrin" sera entendu partout au Québec lors des élections suivantes et il contribuera à balayer l'ancien régime. Les députés [8] de l'opposition se rendent bien compte que ces attaques portent fruit et le prestige de Duplessis s'accroît d'autant. Il est évident aussi que Duplessis souhaite la rupture avec Gouin, rupture qui se consacre d'ailleurs en juin 1936. Peu après, lors d'une réunion du caucus convoquée par Duplessis à Sherbrooke, 35 des 42 députés d'opposition lui donnent leur appui. Duplessis est alors bien en selle comme chef de l'Union nationale.

Les événements se précipitent en cet été 1936. Taschereau démissionne comme premier ministre le 11 juin et Adélard Godbout le remplace. Des élections générales sont convoquées pour le 17 août. Le programme de l'Union nationale, tel qu'exposé par Maurice Duplessis à l'ouverture de la campagne, conserve son allure réformiste. Sans donner beaucoup de précisions, Duplessis s'engage à débarrasser la province des "trusts odieux et exploiteurs" ; mais plusieurs de ses candidats, Hamel et Grégoire en tête, font de ce thème leur cheval de bataille. Duplessis promet aussi un crédit rural provincial pour "sauver l'agriculture" et empêcher l'exode des ruraux vers les villes. En outre, il propose plusieurs réformes politiques qu'il ne réalisera jamais, comme par exemple l'obligation des partis à fournir la liste des souscripteurs à leur caisse, une loi des élections honnêtes et des soumissions publiques pour les contrats donnés par l'État. Par ailleurs, Duplessis consacre une grande partie de sa campagne à dénoncer les scandales du régime Taschereau-Godbout et il ne fait pas de doute que ce thème a un impact énorme sur l'issue du scrutin. Au terme d'une rude campagne, 76 unionistes sont élus contre seulement 14 libéraux ; le chef libéral, Adélard Godbout, est lui-même défait. Au niveau des 
suffrages populaires, le vote libéral chute de 50,2\% qu'il était en 1935 à $41,8 \%$ alors que le vote de l'Union nationale grimpe de $48,7 \%$ à $57,5 \%$. À n'en pas douter, il s'agit d'un véritable renversement électoral. Duplessis et l'Union nationale ont reçu un mandat solide.

\section{Premier mandat, 1936-1939}

$\underline{\text { Retour à la table des matières }}$

En comparant le premier bref mandat de l'Union nationale au long séjour au pouvoir après 1944, il est peut-être tentant de voir deux régimes, deux chefs même, bien différents. En effet, le premier mandat se solde par une véritable débâcle électorale, alors qu'après 1944 Duplessis réussit à consolider son emprise sur le parti et le gouvernement à tel point qu'il gagne quatre élections consécutives, et s'il avait vécu, il y a tout lieu de croire qu'il en aurait remporté une cinquième en 1960. Même en admettant que l'organisation et l'expérience manquent à l'Union nationale au cours des années 1936-1939 et que la conjoncture économique ne lui est guère favorable, les ressemblances entre les deux mandats apparaissent tout aussi frappantes que le sont les contrastes.

[9]

À brève échéance, Duplessis fait de l'Union nationale un parti conservateur et en cela il est sans doute appuyé par la plupart de ses députés et organisateurs qui ne croient pas à un rôle actif Pour l'État. En effet, ce qui leur importe est de prendre le pouvoir, et le garder. Mais plusieurs réformistes bien connus, tels Hamel, Chaloult, Grégoire et Oscar Drouin, défenseurs d'un vigoureux nationalisme économique, rompent tôt avec lui en l'accusant de renier son programme. Ils se mettent à vilipender celui que Hamel qualifie maintenant de "politicien le plus fourbe" qu'il ait jamais rencontré. Qui plus est, ils créent en 1937 un nouveau parti, le parti national. Il n'y a effectivement pas de doute que Duplessis a relégué aux calendes grecques la lutte contre les trusts comme d'ailleurs la plupart des réformes politiques qu'il avait promises. Duplessis aurait même déclaré à Chaloult, quelques semaines après sa victoire : "Un programme, c'est bon avant les élections et les élections sont terminées". Mais il est peut-être vrai aussi, 
comme Mgr Georges Gauthier, archevêque de Montréal, l'a dit, que "la population s'intéresse plus au prix du lait qu'au prix de l'électricité". Et encore Duplessis réussit même à obtenir certaines réductions de tarifs d'électricité dans les villes.

En ce qui concerne les relations entre le gouvernement et les syndicats, celles-ci ne tardent pas à se détériorer. Duplessis fait voter les projets de loi 19 et 20 qui soustraient à l'application de la Loi des salaires raisonnables les travaux publics exécutés pour le compte du gouvernement, interdisent l'atelier fermé et permettent à l'État de modifier unilatéralement les conventions collectives de travail. Puis, en août 1937, 10,000 tisserands, membres de la Confédération des travailleurs catholiques du Canada, déclenchent la grève avec l'appui du clergé. Le gouvernement paraît se ranger du côté du patronat et William Tremblay, ministre du Travail, est qualifié d'«assassin de la classe ouvrière». Mais en vérité il n'y a rien de surprenant à ce que les syndicats aient déjà maille à partir avec Duplessis. Quoiqu'il promettait de défendre l'ouvrier, le chef unioniste n'a jamais tenté de courtiser les syndicats. Les choses ne changeront guère après 1944.

Mais Duplessis ne fait pas qu'attiser des oppositions lors de son premier mandat. D'autres mesures prises par le gouvernement unioniste lui gagnent des partisans, notamment chez les cultivateurs qui, l'orage de 1939 passé, lui manifesteront une loyauté indéfectible. Duplessis exprime en maintes occasions sa philosophie de base, conservatrice, ruraliste : "L'agriculture est un élément de stabilité économique et d'ordre social. Nous devons maintenir et protéger notre base rurale." Et il la concrétise en établissant le crédit agricole et en prenant diverses autres initiatives visant à favoriser la prospérité de l'agriculture.

Le conservatisme de Duplessis se traduit également par une lutte vigoureuse contre la présumée menace communiste au Québec. En ces [10] temps de crise économique et de combats épiques en Europe entre la gauche et la droite - la guerre civile éclate en Espagne en 1936 le clergé catholique s'inquiète de plus en plus de menées subversives dans la province. Le cardinal J.-M.-Rodrigue Villeneuve affirme que le communisme est devenu "une réalité. Le feu est allumé parmi nous et il est urgent de le circonscrire puisqu'on ne peut espérer d'ici longtemps l'éteindre." En octobre 1936, 15,000 fidèles, dont le cardinal, le maire de Québec et le nouveau premier ministre de la province, se ré- 
unissent au Colisée de Québec pour lancer une croisade contre le communisme. Le clergé salue chaleureusement l'adoption, en mars 1937, de la célèbre "loi du Cadenas" et réclame que la législation soit appliquée rigoureusement. Cette lutte se poursuivra tout au long du deuxième mandat de Duplessis.

Bref, au lieu de tenter de tracer le portrait d'un Duplessis sensiblement différent de celui qui reprendra le pouvoir en 1944, il faut peutêtre voir un chef qui jette les assises du support électoral dont il bénéficiera après son retour. Néanmoins, il semble évident qu'au cours des années 1936-1939, Duplessis néglige l'organisation de l'Union nationale de sorte que le parti West nullement préparé à la bataille lorsque le premier ministre convoque brusquement des élections générales à l'automne 1939. Les finances publiques du Québec sont en mauvais état et Ottawa contrôle maintenant les emprunts des provinces. Duplessis cherche donc à fonder sa campagne sur le thème de l'autonomie provinciale, particulièrement financière, et il dénonce ce qu'il appelle le plan fédéral "d'assimilation et de centralisation". En effet, l'élection se fera principalement sur un thème à caractère nationaliste mais ce thème ne sera pas l'autonomie provinciale. On parlera surtout de conscription militaire et l'épouvantail de 1917 hantera à nouveau les esprits. Les ministres québécois à Ottawa, Ernest Lapointe en tête, prétendent que la réélection de Duplessis signifiera un vote de nonconfiance à leur égard et qu'ils devront démissionner. Sans leur présence au cabinet, qui empêcherait un gouvernement anglophone de recourir une nouvelle fois à la conscription? De son côté, Adélard Godbout, chef libéral provincial, s'engage lui-même à démissionner si un seul Canadien français est mobilisé contre son gré sous un régime libéral. Plus tard, les partisans de Duplessis dénonceront ce chantage et prétendront que le Québec a voté sous la peur. Mais il reste que la majorité des électeurs jugent que les ministres fédéraux du Québec constituent une meilleure garantie que Duplessis contre une éventuelle conscription pour service outremer. Les résultats du scrutin sont catastrophiques pour l'Union nationale qui ne fait élire que 15 députés contre 70 libéraux. Par ailleurs, le vote unioniste dégringole de 57,5\% en 1936 à 39,2\% en 1939. 


\section{Séjour dans l'opposition, 1939-1944}

$\underline{\text { Retour à la table des matières }}$

Certains analystes comme Leslie Roberts estiment que Duplessis, en déclenchant des élections en 1939, a commis "l'unique erreur de jugement de sa carrière". Après coup, cependant, il était peut-être mieux que Duplessis soit défait car, par la suite, il a pu assister, comme chef de l'opposition, à l'accomplissement de ses sombres prophéties concernant la conscription et l'autonomie provinciale. En effet, afin de poursuivre l'effort de guerre, le gouvernement fédéral entreprend de centraliser les finances publiques ainsi que les pouvoirs économiques et sociaux. Mais même si les prédictions de Duplessis se sont réalisées, on ne peut nullement conclure qu'il a souhaité la défaite en 1939 ; celle-ci lui a paru d'ailleurs fort amère.

Au lendemain de sa défaite, l'Union nationale, comme d'autres mouvements issus de la crise et qui avaient déçu leurs partisans, semble vouée à la disparition. Duplessis paraît un chef éphémère, un simple trait d'union de l'histoire, et un groupe de conservateurs cherche déjà à l'évincer de la direction du parti. Un Canada anglais triomphant affirme que le Québec a voté pour l'unité nationale et pour l'effort de guerre. Puis, en 1940, la Commission Rowell-Sirois, qui avait enquêté sur les relations entre le Dominion et les provinces, remet son rapport. L'une des recommandations propose le retrait des provinces, en faveur d'Ottawa, du champ des impôts directs en retour de subventions fédérales et du paiement des dettes existantes. Duplessis, qui avait contesté le mandat même de la Commission au moment où elle fut créée en 1937, déclare alors que le rapport constitue "l'apothéose de la dictature de l'argent sur les ruines de l'autonomie, gardienne de nos traditions les plus chères et de nos droits les plus sacrés". En conférence de presse, il soutient le droit du Québec à tous les revenus que lui accorde la Constitution de 1867. Godbout, cependant, n'a guère le choix : en 1942 il sera forcé de conclure une entente de "location" des impôts provinciaux avec Ottawa. 
En Europe de l'Ouest, seule l'Angleterre résiste encore à l'agresseur nazi. Au Canada, de plus en plus d'anglophones estiment que le pays ne fait pas sa part. Devant la montée du sentiment proconscriptionniste à l'intérieur même de son propre parti, W.L.M. King, premier ministre fédéral, décide de tenir un plébiscite dans lequel on demandera aux électeurs de délier le gouvernement de ses promesses anti-conscriptionnistes antérieures. Au Québec, la Ligue pour la défense du Canada fait campagne pour le "non" et, en effet, le "non" triomphe facilement dans cette province au moment du plébiscite d'avril 1942. Par contre, le reste du Canada vote très majoritairement pour le "oui". De la Ligue sortira le Bloc populaire, parti anticonscriptionniste, anti-impérialiste, autonomiste et réformiste, avec une aile fédérale et une aile provinciale, cette dernière dirigée par André Laurendeau. Une fois de plus, comme en 1935, l'opposition est fragmentée [12] et Duplessis risque de se voir doubler par un autre parti plus nationaliste que le sien. Duplessis a beau accuser le Bloc de diviser les forces politiques anti-libérales et de maintenir Godbout au pouvoir; cette fois-ci, il n'y aura aucune coalition antigouvernementale et trois partis séparés se feront la lutte aux élections provinciales du 8 août 1944. Au cours de leur mandat, les libéraux avaient pris plusieurs mesures importantes mais controversées. Ils avaient accordé le droit de vote aux femmes, rendu l'instruction obligatoire pour les enfants de 6 à 14 ans, adopté des législations touchant la fonction publique et les relations patronales-ouvrières, et étatisé deux compagnies privées d'électricité, la Beauharnois et la Montreal Light, Heat and Power, "les deux enfants cancéreux de 44 ans de régime libéral" comme le disait Duplessis. Le chef unioniste accuse Godbout de vouloir "électrocuter la population" pour lui faire oublier le véritable enjeu de cette consultation électorale : la mollesse du gouvernement libéral provincial devant les centralisateurs fédéraux. Abandonné par Ottawa, le parti libéral s'efforce de convaincre les électeurs que "Battre Godbout, c'est battre King, et battre King, c'est faire le jeu des partisans de la conscription." Pourtant, ce thème n'a plus la même résonance qu'en 1939.

Les résultats de l'élection de 1944 sont assez serrés entre libéraux et unionistes, le Bloc jouant plutôt un rôle de nuisance qui semble avoir favorisé l'Union nationale, notamment dans quelques comtés ruraux de la région montréalaise et des Cantons de l'Est. Les libéraux 
méritent le vote populaire le plus important, 39,5\%, mais à cause de la répartition de leurs voix, et particulièrement de leurs immenses majorités dans certains districts montréalais de langue anglaise, ils ne font élire que 37 députés. L'Union nationale, avec 35,8\% des voix, fait élire 48 candidats. Déjà, sans doute grâce au travail de 1936-1939, elle remporte une majorité des districts ruraux, plus nombreux mais moins peuplés. Maurice Duplessis est à nouveau premier ministre du Québec.

\section{Les assises du pouvoir}

$\underline{\text { Retour à la table des matières }}$

Au sortir de la guerre, le Québec n'est plus ce qu'il était dix ans auparavant. La crise est terminée et l'industrialisation rapide a repris. Grâce à la guerre, l'industrie des métaux et l'industrie chimique, entre autres, connaissent un essor prodigieux ; chose inusitée, la valeur de la production manufacturière augmente à un rythme plus rapide au Québec que chez son voisin, l'Ontario. Néanmoins, il reste que l'Ontario possède une plus grande proportion d'industries hautement techniques et rémunératrices alors que le Québec compte beaucoup d'industries, comme le textile, exigeant une main-d'oeuvre abondante et payant des salaires relativement bas. En outre, avec le développement industriel provoqué par la guerre, le nombre de syndiqués au Québec a doublé, passant de 105,000 [13] qu'il était en 1939 à 209,000 en 1946. La C.T.C.C., réputée moins revendicatrice par les patrons, est en nette perte de vitesse - sa part des effectifs syndiqués dans la province décroît de 37\% qu'elle était en 1936 à seulement 24,2\% en 1946 - tandis que les unions internationales progressent rapidement.

Pendant la décennie qui prend fin en 1941, la population totale de la province s'accroît de 16\%, se chiffrant à la fin de la période à près de 3,300,000 ; au cours des années 1941-1951, le rythme d'augmentation s'accélère et atteint près de $22 \%$. Si la population du Québec ne s'urbanise plus durant les années de crise économique, la croissance industrielle des années 1940 signale une reprise de l'exode rural ; en 1951, donc, plus de 67\% des Québécois demeurent dans les villes. La vocation agricole, l'attachement traditionnel des Canadiens français 
aux travaux des champs deviennent de plus en plus des mythes: 25,2\% de la population vit de l'agriculture en 1941 et à peine 19,5\% y trouve son gagne-pain en 1951. Entre 1938 et 1947, l'agriculture ne produit, en moyenne, que $12,4 \%$ de la valeur nette de la production totale au Québec. Qui plus est, bon nombre d'exploitants agricoles s'occupent jusqu'à six mois par année à des travaux à l'extérieur de la ferme. Décidément, le Québec de Maria Chapdelaine n'existe plus.

Devant pareilles transformations, il peut paraître paradoxal de prétendre que la force de Duplessis réside dans sa défense des grandes valeurs conservatrices - ruralisme, religion, sauvegarde des traditions et laissez-faire économique - auxquelles s'attachent toujours de larges secteurs, et certainement l'élite, de la société québécoise. L'Union nationale continue de venir en aide aux cultivateurs, de défendre le rôle traditionnel de l'Église catholique au Québec et notamment sa présence dans les institutions sociales et d'éducation, et de combattre les "hérétiques" - communistes, socialistes et Témoins de Jéhovah, entre autres. Elle s'efforce de repousser les assauts centralisateurs du gouvernement fédéral en matière fiscale et législative au nom de la préservation d'une société distincte. Et elle favorise la paix sociale pour attirer les investissements privés, créer des emplois et assurer la prospérité économique de la province. Par ailleurs, le parti met sur pied une organisation efficace, et gouvernement et parti pratiquent abondamment le patronage afin de rendre une partie au moins de l'électorat dépendante de l'Union nationale, et donc redevable à elle. La clef de voûte de cette entreprise est Maurice Duplessis lui-même.

Dans le domaine de l'agriculture, le crédit agricole, mis sur pied au cours du premier mandat de l'Union nationale, est poursuivi : entre 1937 et 1955, près de 50,000 cultivateurs en profitent. Puis, en 1945, Duplessis présente lui-même un projet de loi créant l'Office d'électrification rurale, chargé d'aider les coopératives à répandre l'électricité dans les campagnes. Si [14] seulement 20\% des propriétés rurales y ont accès en 1944, près de 90\% possèdent l'électricité en 1955.

L'appui que ces mesures, de même que d'autres initiatives touchant la colonisation et l'amélioration des terres, suscitent dans les campagnes est démesurément amplifié par une carte électorale de plus en plus désuète - et injuste pour les citadins - qui accorde une énorme importance politique aux petites circonscriptions rurales. Ainsi, en 1956, les dix districts électoraux les plus peuplés de la province 
contiennent une moyenne d'environ 70,000 électeurs chacun : ils élisent 6 libéraux, sur les 20 libéraux victorieux dans les élections de cette année-là, ainsi que 4 unionistes. Par contre, les dix plus petits comtés n'ont en moyenne que 8,500 inscrits chacun et neuf d'entre eux envoient un député unioniste à Québec. Ce déséquilibre explique en partie pourquoi l'Union nationale domine l'Assemblée législative, avec 82 sur 92 députés en 1948, 68 sur 92 en 1952, et 72 sur 93 en 1956. Rappelons, cependant, qu'à chacune de ces trois consultations électorales, le parti gouvernemental recueille, quoique de justesse, une majorité absolue des suffrages populaires. Faut-il préciser que l'Union nationale ne s'appuie pas que sur les cultivateurs, que même les circonscriptions ouvrières accordent habituellement une majorité de leurs voix à Duplessis ? En effet, la politique anti-syndicale de l'Union nationale ne semble pas lui être coûteuse au moment des élections, peut-être parce que les syndicats suscitent beaucoup de méfiance en milieu populaire, ou que le parti se rachète ailleurs, ou qu'il n'existe tout simplement pas de choix. De fait, les seuls districts où le parti fait vraiment piètre figure se trouvent dans la région anglophone de l'Ouest de l'Île de Montréal.

La plupart des observateurs du règne de Duplessis soulignent le haut degré de collaboration prévalant entre le gouvernement et le clergé catholique de la province. En effet, après les tensions de l'époque de Taschereau, les relations entre l'Église et Duplessis apparaissent généralement bonnes. D'ailleurs, certains évêques semblent même croire que le retour au pouvoir des libéraux signalerait le début d'une vague de sécularisation. Toutefois, vers la fin du régime Duplessis, les relations entre certains membres du clergé et le gouvernement unioniste se détériorent, quoique les deux autorités continuent d'apprécier les avantages d'une collaboration étroite. D'une part, devant les besoins financiers sans cesse croissants et en l'absence d'octrois statutaires aux institutions sociales et aux écoles, le clergé doit constamment solliciter du gouvernement des subventions spéciales. En retour, les effusions de gratitude affluent. Dans les diocèses des régions nouvelles, les appels se font plus pressants et plus fréquents et les expressions de reconnaissance si chaleureuses que certains évêques - c'est notamment le cas de Mgr J.-A. Desmarais, évêque d'Amos - semblent se comporter en véritables agents de l'Union nationale. Sans [15] doute aussi le clergé approuve-t-il les campagnes que Duplessis mène 
contre les communistes et les Témoins de Jéhovah. Cette répression, tant réprouvée à l'extérieur de la province, suscite généralement l'appui de la majorité des catholiques du Québec. Mais d'autre part, Duplessis, devenu un modèle d'orthodoxie fiscale après les problèmes financiers aigus qu'il a rencontrés entre 1936 et 1939, reconnaît que les institutions gérées par le clergé épargnent des sommes énormes en salaires au Trésor public. Tout compte fait, Duplessis et la majorité du clergé, au-delà de leurs intérêts précis, tiennent un langage semblable, un même discours sur la société.

L'opposition libérale, puis le nouveau gouvernement libéral qui succède au régime unioniste en juin 1960 ont souvent reproché à Duplessis d'avoir pratiqué une autonomie provinciale négative qui consistait simplement à refuser les initiatives fédérales et donc à faire perdre d'importantes sommes d'argent aux citoyens du Québec. Toutefois, le contexte des années 1940 et 1950 est bien différent de celui qui prévaut au début des années 1960. Dans la foulée d'une désastreuse crise économique et d'une deuxième guerre mondiale qui paraît exiger la mobilisation de toutes les ressources canadiennes, le gouvernement fédéral tente, avec beaucoup de succès, de centraliser les pouvoirs fiscaux et même législatifs au Canada. Dans l'après-guerre, pour des raisons à la fois sociales, économiques et politiques, les gouvernements de King et de Louis Saint-Laurent poursuivent cet effort en instituant plusieurs nouveaux programmes sociaux, en concluant des accords de location des champs fiscaux avec toutes les provinces à l'exception du Québec, et en mettant sur pied un important programme de financement aux universités canadiennes. Duplessis défend la thèse autonomiste traditionnelle et déclare aux centralisateurs fédéraux : "Vous ne crucifierez pas la province de Québec, même sur une croix d'or."

Dans le dossier des relations fédérales-provinciales des années 1944-1959, deux questions jouent le rôle de vedette : les subventions fédérales aux universités, mises sur pied en octobre 195 1, et l'affaire de l'impôt provincial institué par Duplessis en 1954. Dans le cas des octrois aux universités, Duplessis soutient que le projet constitue une "dangereuse usurpation de pouvoirs par le fédéral, dans un domaine fondamental exclusivement réserve aux provinces" et, sauf la première année, il refuse d'accepter les subsides malgré les besoins croissants des universités de la province. Les groupements nationalistes l'ap- 
puient, et même Pierre Elliott Trudeau, un adversaire habituel du régime, condamne l'intervention fédérale au nom du principe d'une claire attribution des responsabilités dans une fédération. Dans le deuxième cas, le gouvernement unioniste décide de lever un impôt provincial sur le revenu égal à $15 \%$ de l'impôt fédéral dont on réclame la déduction. Les nationalistes se rallient à Duplessis et Gérard Filion, pourtant souvent critique, approuve dans Le Devoir : "Québec reprend [16] l'offensive". Ottawa refuse le principe de la déductibilité mais finit par accepter de réduire de 10\% son impôt sur le revenu des particuliers. Évidemment, il est impossible d'évaluer en termes de rentabilité électorale l'impact des campagnes autonomistes de Duplessis tout comme il serait difficile de démontrer que les positions de Duplessis dans ce domaine coûtent beaucoup d'appuis à l'Union nationale. Il reste, cependant, que les groupements nationalistes appuient la cause de l'autonomie dont Duplessis est le porte-étendard tandis qu'ils reprochent aux libéraux de ne pas donner des garanties suffisantes dans ce domaine.

En matière économique, par contre, ce ne sont pas, tant s'en faut, des valeurs nationalistes qui déterminent la politique du régime. De fait, les critiques dénoncent la contradiction flagrante entre le nationalisme politique pratiqué avec ardeur par Duplessis et les concessions importantes qu'il fait afin d'attirer des investisseurs étrangers. De vastes étendues de terres de la couronne, notamment au NouveauQuébec, sont cédées à des exploitants américains, les redevances sont maintenues à des taux que les libéraux décrient comme étant exagérément généreux pour les compagnies et des commutations de taxes sont accordées. Par ailleurs, la santé financière de la province permet de garder un niveau de taxation relativement bas. En ce qui concerne les relations de travail, le gouvernement, par les législations qu'il adopte et par la façon dont il les applique, cherche à favoriser la "paix sociale". Les contrôles qu'il impose expliquent en partie pourquoi le Québec ne connaît que 35 grèves en moyenne par année, entre 1945 et 1959, impliquant la perte de 335,000 jours de travail, alors que l'Ontario enregistre des pertes moyennes de 700,000 jours de travail par année. En raison du contexte de prospérité, au Québec comme sur le plan international, les investissements affluent vers les secteurs primaire et secondaire. 
La question du développement économique du Québec à cette époque soulève de nombreuses controverses. Assurément, l'économie québécoise connaît un essor prodigieux et on peut aligner les chiffres pour en faire une démonstration convaincante. Mais il semblerait que les écarts de 1939 entre le Québec et l'Ontario, en ce qui concerne la performance économique des deux provinces, persistent en faveur de l'Ontario durant les années 1940 et 1950. Par ailleurs, il est significatif de noter que les plus fortes augmentations de l'embauche se font, non pas dans les secteurs primaire et secondaire, mais bien dans le secteur tertiaire, particulièrement dans les transports, le commerce et les services. Mais malgré certaines faiblesses de la structure économique, le développement rapide de cette période renforce la position du parti gouvernemental et contribue à apaiser les critiques. Contrairement aux tensions qui animent les rapports entre le gouvernement [17] et les syndicats, les relations entre l'État et le monde des affaires demeurent harmonieuses.

Certes, le conservatisme de Maurice Duplessis plaît à de larges et influentes fractions de la population québécoise. Mais on ne saurait minimiser l'importance de l'organisation politique et du patronage quand il s'agit de mobiliser le vote en faveur de l'Union nationale. À partir de 1944, Joseph-D. Bégin, ministre de la Colonisation, est responsable de l'organisation provinciale et Gérald Martineau, nommé conseiller législatif, occupe le poste de trésorier depuis 1946. Ceux qui contribuent à la caisse électorale sont, en gros, toutes les maisons d'affaires de la province. Le gouvernement accorde les contrats de la voirie, sans appel d'offres, à des souscripteurs de la caisse. Le patronage devient un instrument de chantage, voire d'intimidation, une véritable pieuvre qui s'étend à tous les domaines de l'administration provinciale. Pour Jean Lesage, devenu chef du parti libéral en 1958, "tout le monde en profitait, jusqu'au niveau de l'électeur avec son voyage de grenotte la veille de l'élection, pour mettre sur la vase de son entrée de ferme". Et la manne est susceptible de disparaître si les électeurs préfèrent comme député un candidat de l'opposition. Duplessis lui-même affirme, lors d'une assemblée politique dans Verchères en 1952 : "Je vous avais avertis en 1948 de ne pas élire le candidat libéral. Vous ne m'avez pas écouté. Malheureusement votre comté n'a pas obtenu les subventions, les octrois qui auraient pu le rendre plus heureux. J'espère que la leçon aura servi." Hélas ! Les électeurs s'obstineront une fois 
de plus et n'éliront l'unioniste qu'en 1956. Avec les années, cette conception "traditionnelle" de la politique, où le patronage tant sur le plan individuel qu'au niveau collectif joue un grand rôle, sera de plus en plus critiquée par une minorité grandissante d'électeurs. Néanmoins, il semble évident que le favoritisme pratiqué par l'Union nationale a contribué à assurer les votes de ceux qui en profitaient.

\section{Le duplessisme contesté}

$\underline{\text { Retour à la table des matières }}$

Sur le plan électoral, le support donné à l'Union nationale demeure remarquablement stable entre 1948 et 1956. Même en 1960, alors que Duplessis et son successeur, Paul Sauvé, sont disparus et que le troisième chef unioniste en moins d'un an, Antonio Barrette, est aux prises avec des dissensions aiguës au sein de son propre parti, l'Union nationale ne perd qu'un maigre $5 \%$ du vote populaire. Et pourtant les libéraux, avec un nouveau chef dynamique, vantent leur "équipe du tonnerre" et proposent un programme de réformes autour du slogan "C'est le temps que ça change". Certes, les adversaires de Duplessis deviennent plus nombreux et volubiles dans les dernières années du régime mais leur impact sur la base [18] électorale de l'Union nationale apparaît relativement mince. Il se peut bien que la forte opposition dont Duplessis a été constamment l'objet dans les milieux intellectuels explique une tendance, chez des analystes sympathiques à ces éléments, à accorder aux groupements anti-duplessistes une importance exagérée.

En voulant se vanter de l'autorité dont il jouissait, Duplessis aurait souvent déclaré que "les évêques mangent dans ma main". Malgré la collaboration manifeste entre l'Église et le gouvernement unioniste que nous avons déjà soulignée, l'harmonie est loin d'être parfaite. L'attitude du clergé à l'occasion de la célèbre grève de l'amiante en 1949 les évêques vont jusqu'à ordonner des quêtes pour les familles des grévistes - déplaît assurément à Duplessis. Lorsque Mgr Joseph Charbonneau, archevêque de Montréal et ardent défenseur des ouvriers, est obligé de démissionner quelques mois plus tard, certains y voient la main vengeresse de Duplessis; pourtant le consensus actuel veut que 
d'autres raisons aient déterminé son renvoi. Le successeur de Mgr Charbonneau, Mgr Paul-Émile Léger, maintient des relations correctes avec le gouvernement, mais à l'encontre de certains de ses collègues, n'a pas l'habitude de venir mendier à Québec. D'autres prêtres font figure, à titre individuel, de critiques de Duplessis. C'est le cas notamment du Père Georges-Henri Lévesque, doyen de la Faculté des Sciences sociales de l'Université Laval, et des abbés Gérard Dion et Louis O'Neill, auteurs d'un texte paru en août 1956 dénonçant le scandale de l'immoralité politique au Québec.

Les syndicats constituent sans doute la force d'opposition la plus constante au régime même si certaines unions, comme la Fédération provinciale du Travail du Québec, préfèrent composer avec le gouvernement en retour de faveurs. En 1949, Duplessis tente d'imposer des contrôles draconiens aux syndicats dans la province par le projet de loi numéro 5. Il interdit l'atelier fermé et détermine les conditions d'exercice du droit de grève. Par ailleurs, il va jusqu'à stipuler que tout syndicat qui permet à des "communistes" d'occuper des postes d'organisateur ou d'officier perdra le certificat qu'il détient de la Commission des relations ouvrières et à toutes fins utiles, son droit de négocier avec l'employeur. Les syndicats s'y opposent avec acharnement et l'Église réprouve aussi le projet. Duplessis le retire alors mais il réussira à implanter, morceau par morceau, plusieurs de ses dispositions, notamment par le truchement des projets de loi 19 et 20 que la législature adopte, en 1954, avec effet rétroactif jusqu'en 1944. La C.T.C.C., maintenant aguerrie par la grève de l'amiante et par une série d'autres grèves en 1952 où la police sera utilisée fréquemment contre les grévistes, fait front commun contre ces mesures législatives avec la Fédération des Unions industrielles du Québec. La F.P.T.Q. et la F.U.I.Q. se fusionnent en 1957 pour former la Fédération du Travail du Québec, [19] centrale ouvrière qui conduira l'interminable grève de Murdochville survenue cette même année.

Le régime Duplessis est aussi pris à partie par une minorité croissante d'intellectuels. Des foyers d'opposition se trouvent, entre autres, au journal Le Devoir à Montréal, à la petite revue Cité libre, animée par Pierre Elliott Trudeau et Gérard Pelletier, à la revue Relations publiée par les Jésuites, et dans les universités, notamment à la Faculté des Sciences sociales de l'Université Laval. Certes, ces critiques ne cessent de condamner les pratiques électorales de l'Union nationale, le 
favoritisme éhonté, et les scandales entourant le régime, dont le plus célèbre a sans doute été celui du gaz naturel de 1958. Mais ils vont beaucoup plus loin car, pour eux, le Québec des années 1950 accuse un retard grave sur les autres provinces canadiennes particulièrement en ce qui a trait au rôle qu'ils estiment que l'État devrait jouer dans une société moderne. Ces intellectuels dénoncent le conservatisme social inhérent à la doctrine de l'autonomie provinciale telle que défendue par Duplessis. Sans verser dans l'anticléricalisme, ils croient que l'État devrait s'impliquer davantage dans les champs de l'éducation et du bien-être social, alors largement sous le contrôle du clergé catholique. Pour ce qui est de l'éducation en particulier, il faudrait revoir les programmes d'enseignement, améliorer la formation des maîtres, et accroître la scolarisation de la population. De fait, Cité libre va jusqu'à réclamer l'établissement d'un ministère de l'Éducation. Dans le secteur économique, Le Devoir soutient que l'État devrait cesser de se ranger carrément avec les patrons dans l'exploitation des ouvriers et qu'il faudrait imposer des conditions beaucoup plus astreignantes aux investisseurs étrangers qui tirent des profits trop élevés des richesses naturelles de la province. Mais pour réaliser ces réformes, l'État québécois aurait besoin d'une fonction publique étendue qui se distinguerait par sa compétence et non par son allégeance au parti au pouvoir. Néanmoins, les forces d'opposition sont divisées et plusieurs adversaires de l'Union nationale ne se sentent guère plus à l'aise face aux libéraux de Lapalme car, estiment-ils, la vieille garde du parti a encore trop d'emprise sur eux. Seul un nouveau chef avec un programme remanié saura aplanir les divergences de vue et regrouper les éléments anti-duplessistes. C'est effectivement ce qui se produira avec la venue de Jean Lesage en 1958.

\section{$* * *$}

Maurice Duplessis meurt subitement le 7 septembre 1959. Avec son slogan, "Désormais", le nouveau leader, Paul Sauvé, laisse entendre que le duplessisme a vécu, mais lui-même est foudroyé par la mort au début de janvier 1960 avant de pouvoir laisser son empreinte sur la société [20] québécoise. Le caucus unioniste finit par désigner Antonio Barrette comme successeur, mais celui-ci ne réussit pas à se faire 
accepter auprès de certains éléments du parti, notamment au niveau de l'organisation. Le 22 juin 1960, l'Union nationale est battue, quoique de justesse, par les libéraux de Lesage, et le Québec s'engage alors dans la période qu'on a convenu d'appeler la "Révolution tranquille". Le gouvernement prendra le contrôle de la plupart des institutions d'enseignement, de santé et de bien-être, il adoptera un nouveau code du travail, il favorisera un certain nationalisme économique, il poursuivra une politique autonomiste agressive en face du gouvernement d'Ottawa, et il luttera contre le patronage et les abus électoraux.

Dans ce climat d'enthousiasme et d'action, les libéraux et leurs sympathisants ont eu tendance à juger sévèrement la période duplessiste comme étant celle de la "Grande noirceur". Leur avènement au pouvoir aurait signifié en quelque sorte un réveil, un dégel, le commencement d'une ère. Il se peut fort bien que le régime Duplessis ait trop duré, que la politique traditionnaliste du chef unioniste soit devenue anachronique par rapport à la société moderne qu'était déjà, à bien des égards, le Québec des années 1950. Il se peut aussi que les libéraux et leur "équipe du tonnerre", arrivés au pouvoir en 1960, n'aient guère eu le choix et qu'ils se soient sentis obligés d'agir à la hâte sur tous les fronts pour permettre au Québec de combler ses retards. Mais les partisans du nouveau gouvernement ne bénéficiaient guère du recul du temps nécessaire pour porter un jugement un tant soit peu impartial sur l'ancien régime ; par ailleurs, ils ne pouvaient pas non plus apprécier les failles que révéleraient immanquablement les réformes qu'ils venaient d'instituer.

Duplessis et son régime continueront de fasciner les observateurs de la société québécoise et les recherches se poursuivront à mesure que d'autres sources documentaires deviendront disponibles et que d'autres monographies seront rédigées. Libérés dans une plus grande mesure des contraintes partisanes si présentes ces dernières années, ils pourront sans doute jeter de nouvelles lumières sur une période complexe dans l'histoire du Québec. 


\section{Notes bibliographiques}

$\underline{\text { Retour à la table des matières }}$

Duplessis a fait l'objet de plusieurs biographies. Pierre Laporte, Le vrai visage de Duplessis (Montréal, 1962) et Leslie Roberts, Le chef : une biographie-politique de Maurice L. Duplessis (Montréal, 1963) contiennent surtout des impressions. Les études de Robert Rumilly, Maurice Duplessis et son temps (Montréal, 1973, 2 vols.) et Conrad Black, Duplessis (Montréal, 1977, 2 vols.) sont beaucoup plus fouillées mais se révèlent, surtout dans le premier cas, assez partiales. L'ouvrage de Bernard Saint-Aubin, Duplessis et son époque (Montréal, 1979), est moins imposant et semble plus impartial. Plusieurs hommes politiques ont parlé de Duplessis dans leur mémoires : voir en particulier René Chaloult, Mémoires politiques (Montréal, 1969), Antonio Barrette, Mémoires (Montréal, 1966), et Georges-Émile Lapalme, Mémoires (Ottawa, 1969-1973, 3 vols.).

Gérard Boismenu traite de la politique économique de Duplessis dans Le duplessisme: politique économique et rapports de force, 1944-1960 (Montréal, 1981) et Kenneth McRoberts et Dale Posgate cherchent à démontrer le retard dans la modernisation politique du Québec dans Quebec : Social Change and Political Crisis (Toronto, 1980, édition révisée). Pour sa part, Jean-Louis Roy, dans La marche des Québécois : le temps des ruptures (1945-1960) (Montréal, 1976) [Livre disponible dans Les Classiques des sciences sociales. JMT.], étudie la gestation des transformations sociales préparant la Révolution tranquille. Les principales grèves sont abordées dans Pierre Elliott Trudeau (éd.), La grève de l'amiante (Montréal, 1956, réédité en 1970) et Jean-Paul Lefebvre et al., En grève! (Montréal, 1963). Louis-Marie Tremblay discute de la pensée syndicaliste dans Le syndicalisme québécois : idéologies de la C.S.N. et de la F.T.Q., 19401970 (Montréal, 1972). Herbert F. Quinn, The Union Nationale : A Study in Quebec Nationalism (Toronto, 1963, révisé et réédité en 1979) présente une histoire politique de la période. Mario Cardinal, Vincent Lemieux et Florian Sauvageau offrent des témoignages fort 
intéressants de contemporains de la période dans Si l'Union nationale m'était contée... (Montréal, 1978) [Livre disponible dans Les Classiques des sciences sociales. JMT.]. Pour les programmes électoraux, voir Jean-Louis Roy, Les programmes électoraux du Québec, tome II. 1931-1966 (Ottawa, 1971). Plusieurs études électorales existent: consulter notamment Paul Cliche, Les élections provinciales dans la province de Québec de 1927 à 1956 (thèse de maîtrise, Québec, 1960, 2 vols.), Jean-Guy Genest, L'élection provinciale québécoise de 1939 (thèse de D.E.S., Québec, 1968), Herbert F. Quinn, The Quebec Provincial Election of 1944 : an Analysis of the Role of the Election in the Democratic Process (thèse de maîtrise, Montréal, 1946) et Vincent Lemieux (dir.), Quatre élections provinciales au Québec, 1956-1966 (Québec, 1969). Robert Boily étudie les [22] élites politiques dans "Les hommes politiques du Québec 1867-1967" (Revue d'histoire de l'Amérique française, XXI, 3a, 1967-1968, pp. 599-634).

La question du patronage est traitée dans Vincent Lemieux et Raymond Hudon, Patronage et politique au Québec, 1944-1972 (Sillery, 1975) [Livre disponible dans Les Classiques des sciences sociales. JMT.]. Pour l'idéologie de Duplessis et de l'Union nationale, lire Richard Desrosiers, "Duplessis et l'idéologie dominante" (R.H.A.F, XXV, 3, décembre 1971, pp. 385-388) et Herbert F. Quinn, "L'idéologie de l'Union nationale, 1936-1976", dans F. Dumont et al., Idéologies au Canada français, 1940-1976 : tome III (Québec, 1981) [Livre disponible dans Les Classiques des sciences sociales. JMT.]. La question de l'autonomie provinciale est abordée dans René Durocher, "Maurice Duplessis et sa conception de l'autonomie provinciale au début de sa carrière politique" (R.H.A.F., XXIII, 1, juin 1969, pp. 1314) et René Durocher et Michèle Jean, "Duplessis et la Commission royale d'enquête sur les problèmes constitutionnels, 1953-1956" (R.H.A.F., XXV, 3, décembre 1971, pp. 337-363). Cameron Nish présente les controverses qui entourent plusieurs aspects du régime Duplessis dans Quebec in the Duplessis Era : Dictatorship or Democracy? (Toronto, 1970). Enfin, René Durocher tente d'expliquer le succès politique de Duplessis dans "Le long règne de Duplessis : un essai d'interprétation" (R.H.A.F., XXV, 3, décembre 1971, pp. 392-396). 


\section{AUTRES BROCHURES}

Conçue par la Société historique du Canada, cette collection a pour objectif de fournir au public en général, aux instituteurs, aux professeurs d'histoire et aux historiens eux-mêmes de courtes études sur quelques problèmes particuliers de l'histoire du Canada. Rédigées en anglais ou en français, ces brochures, traduites, sont disponibles dans les deux langues. On les obtient en s'adressant au Trésorier, Société historique du Canada, Archives publiques du Canada, Ottawa, K1A 0N3. Les membres de la Société reçoivent, à titre gracieux et sur publication, un exemplaire de chaque nouvelle brochure. Ces brochures se vendent \$1.25 l'unité. Une remise de 25\% est consentie aux libraires.

Voici la liste des brochures parues à ce jour :

1. C.P. Stacey, La Frontière sans défense : le mythe et la réalité.

2. G.F.G. Stanley, Louis Riel : patriote ou rebelle.

3. Guy Frégault, La Société canadienne sous le régime français.

4. W.S. MacNutt, L'Émergence des provinces maritimes, 17131784.

5. A.L. Burt, Guy Carleton, Lord Dorchester, 1724-1808.

6. $\quad$ Marcel Trudel, Le Régime seigneurial.

7. F.H. Soward, Le Ministère des Affaires extérieures et l'autonomie au Canada, 1899-1939.

8. F.H. Underhill, Les Partis politiques canadiens.

9. W.L. Morton, L'Ouest et la Confédération, 1857-1871.

10. G.O. Rothney, Terre-Neuve : son histoire.

11. Fernand Ouellet, Louis-Joseph Papineau : Un être divisé.

12. D.C. Masters, La Réciprocité, 1846-1911. 
13. Michel Brunet, Les Canadiens el les débuts de la domination britannique, 1760-1791.

14. T.J. Oleson, Les Scandinaves en Amérique.

15. P. B. Waite, La Conférence de Charlottetown.

16. Roger Graham, Arthur Meighen.

17. J. Murray Beck, Joseph Howe anticonfédérafiste.

18. W.J. Eccles, Le Gouvernement de la Nouvelle-France.

19. Paul G. Cornell, La Grande Coalition.

20. W.M.Whitelaw, La Conférence de Quéhec.

21. J.-C. Bonenfant, Les Canadiens français et la Confédération.

22. Helen I. Cowan, L'Immigration britannique avant la Confédération.

23. Alan Wilson, Les Réserves du clergé du Haut-Canada.

24. Richard Wilbur, Le Gouvernement Bennett, 1930-1935.

25. R.A. Preston, La Politique de défense et le développement de la nation canadienne.

26. Lewis H. Thomas, Les Territoires du Nord-Ouest, 1870-1905.

27. Eugene A. Forsey, Le Mouvement ouvrier au Canada, 18121902.

28. Irving Abella, Le Mouvement ouvrier au Canada de 1902 à 1960.

29. Joseph Levitt, Henri Bourassa - Critique catholique.

30. Bruce G. Trigger, Les Indiens et l'âge hérö̈que de la NouvelleFrance.

31. R.C. Macleod, La Police à cheval au Nord-Ouest, 1873-1919.

32. J.M.S. Careless, L'Expansion des villes canadiennes avant 1914.

33. lan MacPherson, Le mouvement coopératif dans les Prairies, 1900-1955.

34. Alan F.J. Artibise, L'Expansion urbaine dans les Prairies, 18701939. 
35. Richard Jones, Duplessis et le gouvernement de l'Union nationale.

36. Ernest R. Forbes, Certains aspects du régionalisme dans les provinces maritimes, 1867-1927.

\section{Fin du texte}

